\title{
A quantum magnetic analogue to the critical point of water
}

\author{
J. Larrea Jiménez, ${ }^{1,2}$ S. P. G. Crone,${ }^{3}$ E. Fogh, ${ }^{2}$ M. E. Zayed,${ }^{4}$ R. Lortz,${ }^{5}$ E. \\ Pomjakushina, ${ }^{6}$ K. Conder ${ }^{6}$ A. M. Läuchli, ${ }^{7}$ L. Weber ${ }^{8}$ S. Wessel,${ }^{8}$ A. Honecker, ${ }^{9}$ \\ B. Normand, ${ }^{10,2}$ Ch. Rüegg, ${ }^{10,11,2,12}$ P. Corboz ${ }^{3}$ H. M. Rønnow, ${ }^{2}$ and F. Mila ${ }^{2}$ \\ ${ }^{1}$ Institute of Physics, University of São Paulo, CEP 05508-090, São Paulo, SP, Brazil \\ ${ }^{2}$ Institute of Physics, Ecole Polytechnique Fédérale de Lausanne (EPFL), CH-1015 Lausanne, Switzerland \\ ${ }^{3}$ Institute for Theoretical Physics and Delta Institute for Theoretical Physics, \\ University of Amsterdam, Science Park 904, 1098 XH Amsterdam, The Netherlands \\ ${ }^{4}$ Department of Physics, Carnegie Mellon University in Qatar, Education City, PO Box 24866, Doha, Qatar \\ ${ }^{5}$ Department of Physics, Hong Kong University of Science and Technology, Clearwater Bay, Kowloon, Hong Kong \\ ${ }^{6}$ Laboratory for Multiscale Materials Experiments, \\ Paul Scherrer Institute, CH-5232 Villigen-PSI, Switzerland. \\ ${ }^{7}$ Institut für Theoretische Physik, Universität Innsbruck, A-6020 Innsbruck, Austria \\ ${ }^{8}$ Institut für Theoretische Festkörperphysik, JARA-FIT and JARA-HPC, \\ RWTH Aachen University, 52056 Aachen, Germany \\ ${ }^{9}$ Laboratoire de Physique Théorique et Modélisation, CNRS UMR 8089, \\ CY Cergy Paris Université, 95302 Cergy-Pontoise Cedex, France \\ ${ }^{10}$ Paul Scherrer Institute, CH-5232 Villigen-PSI, Switzerland \\ ${ }^{11}$ Institute for Quantum Electronics, ETH Zürich, CH-8093 Hönggerberg, Switzerland \\ ${ }^{12}$ Department of Quantum Matter Physics, University of Geneva, CH-1211 Geneva 4, Switzerland
}

(Dated: October 1, 2020)

At the familiar liquid-gas phase transition in water, the density jumps discontinuously at atmospheric pressure, but the line of these firstorder transitions defined by increasing pressures terminates at the critical point [1], a concept ubiquitous in statistical thermodynamics [2]. In correlated quantum materials, a critical point was predicted [3] and measured [4, 5] terminating the line of Mott metal-insulator transitions, which are also first-order with a discontinuous charge density. In quantum spin systems, continuous quantum phase transitions (QPTs) [6] have been investigated extensively [7-11], but discontinuous QPTs have received less attention. The frustrated quantum antiferromagnet $\mathrm{SrCu}_{2}\left(\mathrm{BO}_{3}\right)_{2}$ constitutes a near-exact realization of the paradigmatic Shastry-Sutherland model [12-14] and displays exotic phenomena including magnetization plateaux [15], anomalous thermodynamics [16] and discontinuous QPTs [17]. We demonstrate by high-precision specific-heat measurements under pressure and applied magnetic field that, like water, the pressure-temperature phase diagram of $\mathrm{SrCu}_{2}\left(\mathrm{BO}_{3}\right)_{2}$ has an Ising critical point terminating a first-order transition line, which separates phases with different densities of magnetic particles (triplets). We achieve a quantitative explanation of our data by detailed numerical calculations using newly-developed finitetemperature tensor-network methods [16, 18-20]. These results open a new dimension in understanding the thermodynamics of quantum magnetic materials, where the anisotropic spin inter- actions producing topological properties [21, 22] for spintronic applications drive an increasing focus on first-order QPTs.

Water boils at $100^{\circ} \mathrm{C}$ at ambient pressure. At this liquid-gas transition the density jumps dramatically, i.e. the transition is first-order. Under an applied pressure, the line of first-order transitions in $(P, T)$ terminates at $P_{c}=221 \mathrm{bar}$ and $T_{c}=374^{\circ} \mathrm{C}$ (Fig. 1 1 ), "the critical point of water," where liquid and vapour become a single phase [1. Because their difference is defined not by a change in symmetry but by a scalar, the particle density, which can take two values, the critical point is in the Ising universality class. Although the first-order line has no critical properties, its termination point (a continuous transition) does 2. In the supercritical regime, meaning around and above the critical point, there is no transition and indeed one may proceed continuously from liquid to vapour without ever undergoing one.

In quantum matter with active charge degrees of freedom, a critical point (which we distinguish from the "critical endpoint" 23]) terminates the Mott metal-insulator transition line [3 [5]. Deep in the insulating phase, systems with only spin degrees of freedom provide theorists with a fertile avenue for realizing quantum many-body models, including those with exact solutions or ground states, and experimentalists with an unparalleled platform for probing their properties on truly macroscopic lengthscales. Continuous QPTs and quantum criticality [6] have been controlled by pressure [7, 8, applied magnetic field [9, 10], and disorder [11]. Here we control both pressure and field to investigate a discontinuous QPT and provide the first observation of critical-point physics in a pure spin system. 

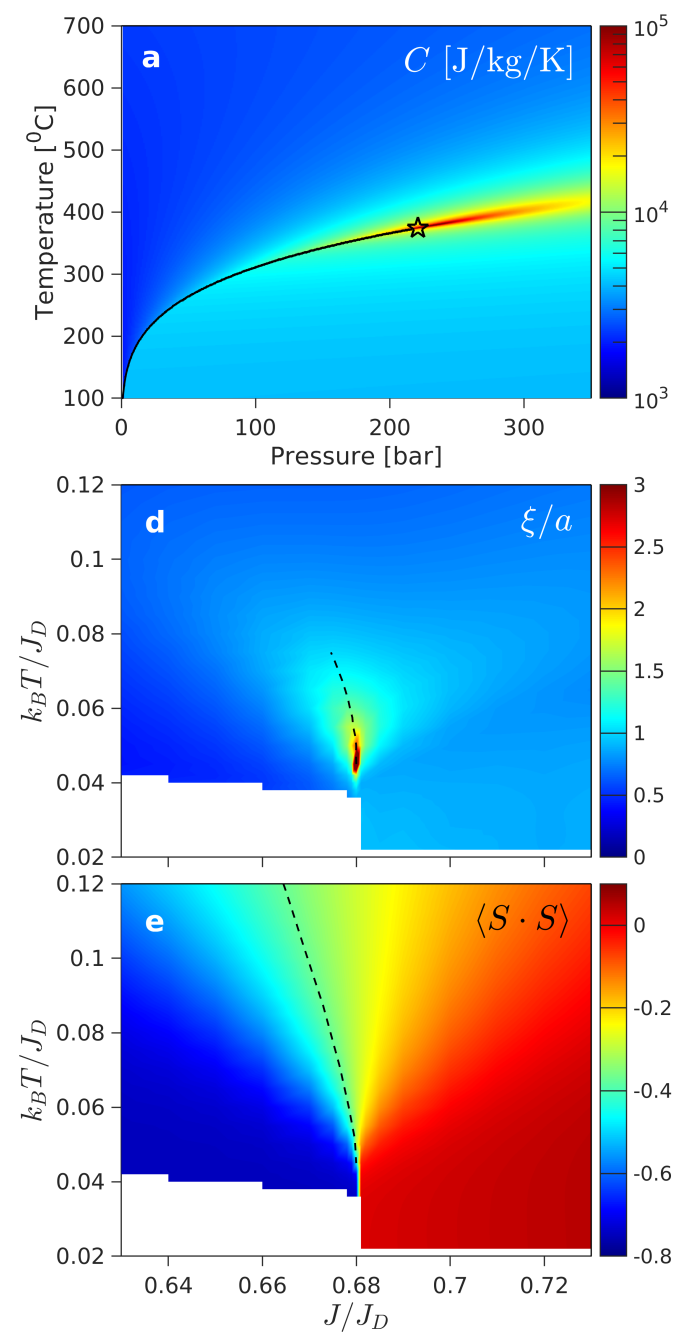
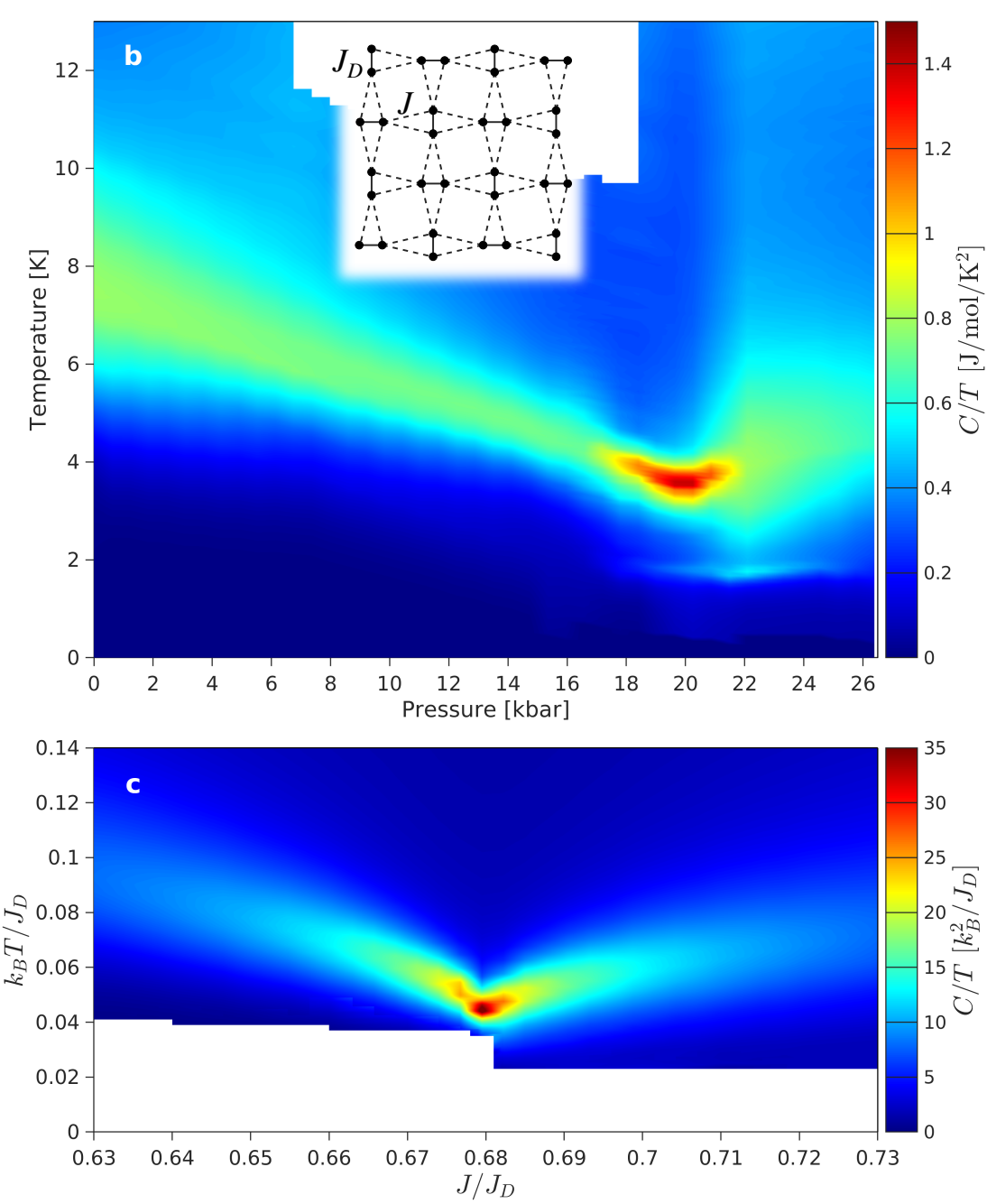

FIG. 1. Specific heat of water, of $\operatorname{SrCu}_{2}\left(\mathrm{BO}_{3}\right)_{2}$ and of the Shastry-Sutherland model. a, Specific heat of water, $C_{p}(P, T) / T$, shown as a function of pressure and temperature. The black line marks the first-order phase boundary separating liquid (lower left) from vapour (upper left) and the black star marks the critical point. b, Experimental data for the specific heat, $C_{p}(P, T) / T$, of $\mathrm{SrCu}_{2}\left(\mathrm{BO}_{3}\right)_{2}$. Below $18 \mathrm{kbar}$ is the dimer product phase and above 20 kbar is the plaquette phase. The two lines of maxima meet at the critical point at approximately 19 kbar and $3.3 \mathrm{~K}$. The inset shows the orthogonal dimer geometry of the Shastry-Sutherland model, which is realized by the $\mathrm{Cu}^{2+}$ ions $(S=1 / 2)$ in $\mathrm{SrCu}_{2}\left(\mathrm{BO}_{3}\right)_{2}$. c, $\mathrm{Analogous}$ data obtained by iPEPS calculations with $D=20$ performed for the Shastry-Sutherland model with different values of the coupling ratio, $J / J_{D}$, which in $\mathrm{SrCu}_{2}\left(\mathrm{BO}_{3}\right)_{2}$ was shown to be an approximately linear function of the applied pressure [17. d, Correlation length, $\xi$, obtained by iPEPS with $D=20$ and expressed in units of the lattice constant, $a$. $\xi$ becomes large only at the finite-temperature critical point; the dashed black line shows the locus of maxima of $\xi\left(J / J_{D}\right)$ at each fixed temperature, which we terminate when $\xi / a<1$. e, Dimer spin-spin correlation function, $\left\langle\vec{S}_{i} \cdot \vec{S}_{j}\right\rangle$, showing a discontinuity with $J / J_{D}$ at low temperatures but continuous behaviour throughout the supercritical regime. The dashed black line, the equivalent of the critical isochore in water, shows the locus of points where this order parameter is constant at its critical-point value, $\left\langle\vec{S}_{i} \cdot \vec{S}_{j}\right\rangle=-0.372(30)$.

Among the key magnetic quantum materials, $\mathrm{SrCu}_{2}\left(\mathrm{BO}_{3}\right)_{2}$ 24] has drawn attention because of the extreme frustration of its orthogonal-dimer geometry (inset, Fig. 1p). This realizes a $S=1 / 2$ Heisenberg model formulated by Shastry and Sutherland [12] because it has an exact ground state, a product of dimer singlets, for all small and intermediate interaction ratios $\left(J / J_{D}<0.675[14]\right)$. While the frustration is manifest in many unusual phenomena 15, 16, 25, our interest in the Shastry-Sutherland model lies in the presence of two QPTs, from the dimer phase to a plaquette phase at $J / J_{D}=0.675(2)$ and thence to an ordered Néel antiferromagnet $(\mathrm{AF})$ at $J / J_{D}=0.765(15)$ [14; our interest in $\mathrm{SrCu}_{2}\left(\mathrm{BO}_{3}\right)_{2}$ lies in the remarkable fact that an applied hydrostatic pressure acts to control $J / J_{D}$, revealing both transitions at respective pressures of 

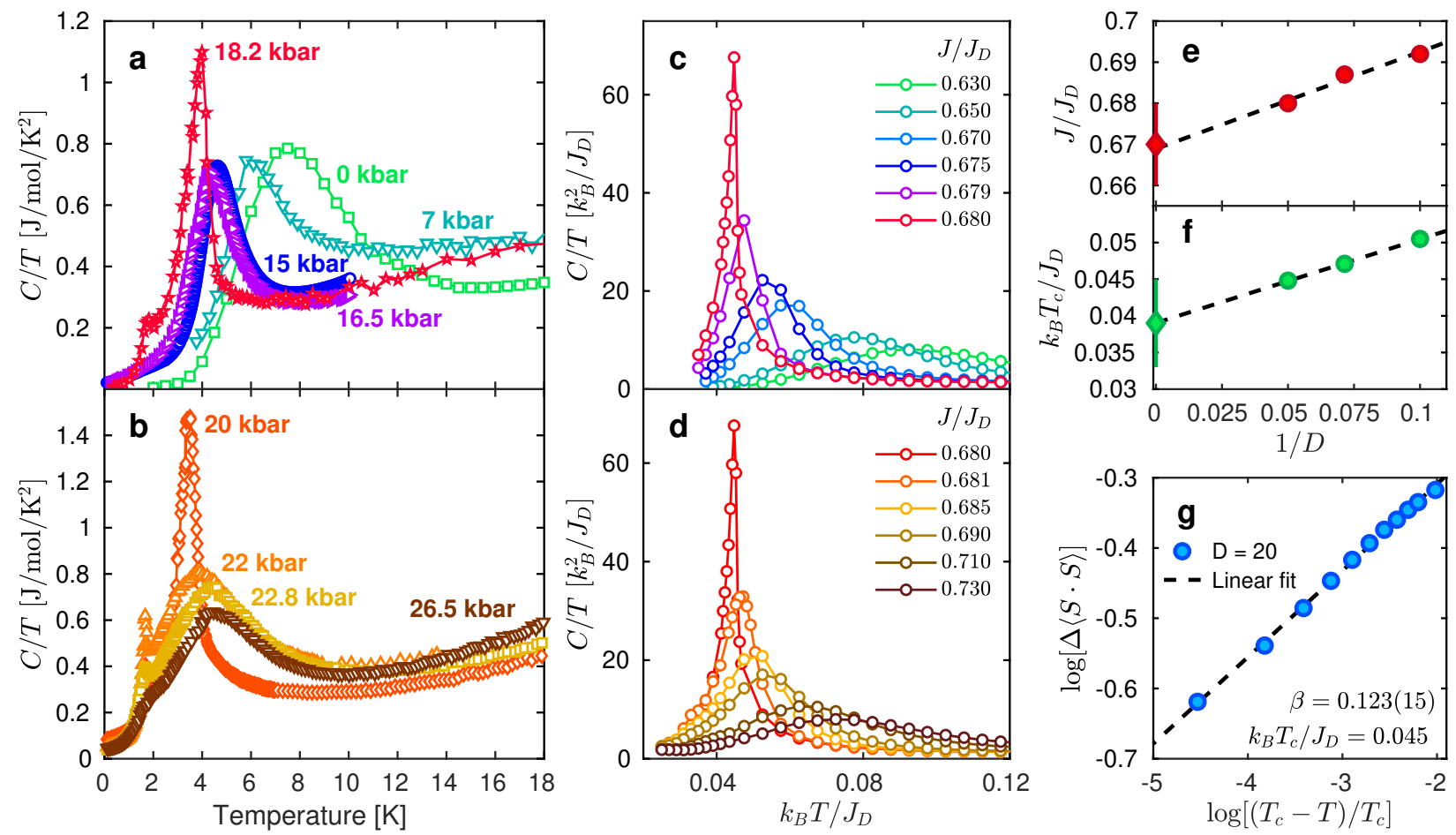

FIG. 2. Specific heat for different pressures at zero magnetic field. a, $C_{p}(T) / T$ measured for applied pressures from 0 to $18.2 \mathrm{kbar}$ and $\mathbf{b}$ from 20 to $26.5 \mathrm{kbar}$. The temperature of the broad peak observed at most pressures $(P \leq 15 \mathrm{kbar}$ and $P \geq 22$ kbar) drops to a finite minimum value at the QPT, where $C_{p}(T) / T$ displays only a tall and extremely narrow peak. c, $\mathbf{d}$, Analogous data obtained by $D=20$ iPEPS calculations performed for the Shastry-Sutherland model in the dimer (c) and plaquette (d) phases using plaquette-based tensors (Methods section). The evolution of peak heights with proximity to the critical coupling ratio, illustrated clearly in the numerical data, is less apparent in experiment. We note that the phonon contribution $\left(C_{p}(T) / T \propto T^{2}\right)$ to the measured specific heat becomes appreciable at higher temperatures; while this can be subtracted for accurate fitting [16, our focus here is on the peak positions at and below $6 \mathrm{~K}$. e, Convergence of the critical coupling ratio obtained in finite-temperature iPEPS calculations as a function of $1 / D$; the extrapolated value of $0.67(1)$ agrees well with the zero-temperature value 14. f, Convergence of the critical temperature as a function of $1 / D$, leading to the estimate $k_{B} T_{c} / J_{D}=0.039(6)$. g, Critical exponent, $\beta=0.123(15)$, of the discontinuity, $\Delta\left\langle\vec{S}_{i} \cdot \vec{S}_{j}\right\rangle$, in the dimer spin-spin correlation function, demonstrating consistency with the $2 \mathrm{D}$ Ising exponent, $\beta=1 / 8$.

approximately $19[17$ and 27 kbar 26 .

The finite-temperature critical point has been discussed theoretically in a two-dimensional (2D) Heisenberg spin model, the "fully frustrated bilayer" 27. In this geometry, which has no known materials analogue, spin pairs (with coupling $J_{\perp}$ ) are arranged vertically on a square lattice with equal couplings $\left(J_{\|}\right)$to both spins of all four dimer neighbours, and the ground state jumps discontinuously from exact dimer singlets to exact triplets at $J_{\perp} / J_{\|}=2.315$. With increasing temperature, the discontinuity in triplet density reduces until the line of first-order transitions terminates at a critical point, in the $2 \mathrm{D}$ Ising universality class, when $k_{B} T_{c} \simeq 0.52 J_{\|}[27$. The connection [28] between the two fully frustrated geometries (bilayer and Shastry-Sutherland) suggests that they may share similar critical-point physics. Although the total spin of each dimer is not a good quantum number in the Shastry-Sutherland case, and hence it may not have the same large $T_{c}$ as the bilayer, a discontinuity in the average dimer spin-spin correlation is already well known at the dimer-plaquette QPT.

To investigate the critical point in $\mathrm{SrCu}_{2}\left(\mathrm{BO}_{3}\right)_{2}$, we perform high-precision measurements of the specific heat using an a.c. calorimetry technique 29. As described in the Methods section, large single crystals of $\mathrm{SrCu}_{2}\left(\mathrm{BO}_{3}\right)_{2}$ were grown by a floating-zone method. Samples of masses up to $36 \mathrm{mg}$ were cut, patterned with metallic strips for calorimetry and mounted in a clamp cell allowing hydrostatic pressures up to $26.5 \mathrm{kbar}$ and in applied magnetic fields up to $9 \mathrm{~T}$. Details of our a.c. measurement procedures are provided in the Methods section.

Starting with zero magnetic field, the pressure-induced evolution of the specific heat, shown as $C_{p}(T) / T$, is illustrated in Fig. 1 1 . As quantified in Fig. 2 a,$C_{p}(T) / T$ at low pressures shows an exponential rise to a broad maximum at a temperature, $T_{\max }$, that tracks the gap to the triplon or bound-triplon excitations of the dimer phase 16]. With increasing $P$, this peak moves gradually lower and becomes proportionately narrower, but between 18 and $20 \mathrm{kbar}$ it becomes extremely narrow and attains a 

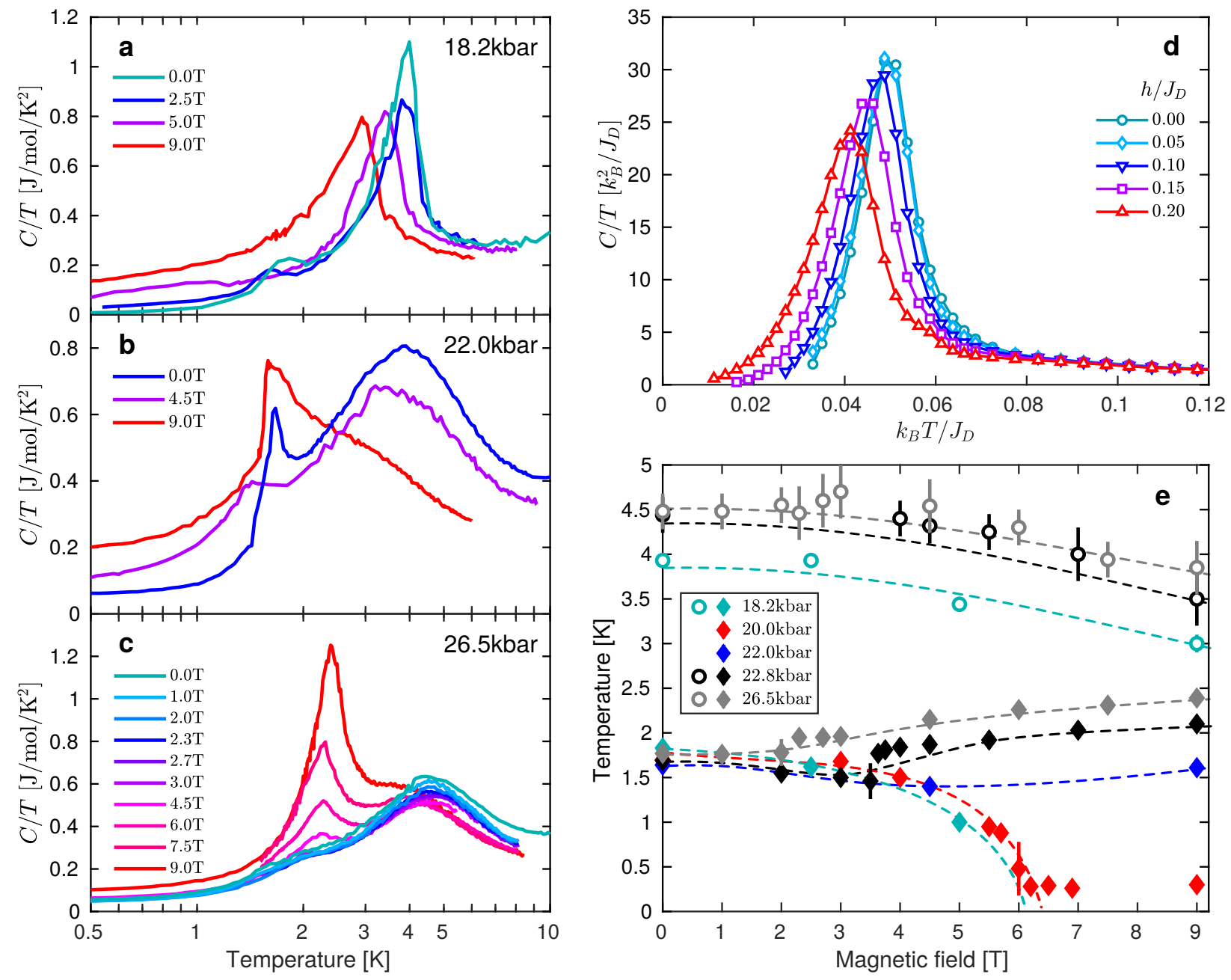

FIG. 3. Evolution of the specific heat with magnetic field for pressures around the QPT. a, $C_{p}(T) / T$ at $18.2 \mathrm{kbar}$ for four different applied fields up to $\mu_{0} H=9 \mathrm{~T}$. The peak due to the critical point retains its sharp nature and moves only slightly downwards in position and height with increasing field. $\mathbf{b}, C_{p}(T) / T$ at 22.0 kbar for three different applied fields, showing initial field-induced suppression of the low-temperature transition followed by a dramatic change in shape to a sharp low- $T$ peak with no broad hump at higher energies. c, $C_{p}(T) / T$ at $26.5 \mathrm{kbar}$, illustrating the field-induced emergence of the new, sharp peak at $9 \mathrm{~T}$, which is suggestive of the transition to the AF phase. $\mathbf{d}, C_{p}(T) / T$ computed by iPEPS at a coupling ratio, $J / J_{D}=0.686$, close to the critical point for $D=14$, for a range of applied magnetic fields. As in panel a, the sharp peak due to the critical point undergoes only a minor field-induced suppression of its position and height, while its width is unaltered. For the $g$-factor of $\mathrm{SrCu}_{2}\left(\mathrm{BO}_{3}\right)_{2}$, a field $h / J_{D} \equiv g \mu_{B} \mu_{0} H / J_{D}=0.2$ corresponds to approximately 11 T. e, Characteristic field and temperature scales revealed by our full set of specific-heat measurements, which separate into high- $T$ features around 4 $\mathrm{K}$ and low- $T$ features around and below $2 \mathrm{~K}$. The high- $T$ feature is the broad maximum, which at the critical point evolves into the sharp peak. The low- $T$ feature at the lower pressures is the small peak due to the thermal Ising transition out of the plaquette phase, which can be suppressed to $T=0$ by the applied field. At the higher pressures this feature changes into the strong peaks arising from the thermal transition of the AF phase, which is favoured by the applied field.

much higher peak value (Figs. 1 b and 2a), bearing all the characteristics of a continuous phase transition, with diverging observables. After reaching a lowest measured value of $3.4 \mathrm{~K}$ at $P=20 \mathrm{kbar}, T_{\max }$ rises with increasing pressure and the peak broadens again (Fig. $2 \mathrm{~b}$ ), indicating that the singular behaviour has terminated.

A second small peak appears around $2 \mathrm{~K}$ for $P \geq 18$ kbar (Figs. 1b and 2a) and persists to our upper pressure limit. We expect that this peak corresponds to the thermal transition out of the plaquette phase and provide a detailed discussion below. Our key observation is that the critical point occurs at a temperature well above the plaquette transition, consistent with its interpretation as the termination of a line of first-order transitions.

To model these thermodynamic results we use the method of infinite projected entangled pair states (iPEPS), which are a variational tensor-network Ansatz for the representation of a quantum state on an infinite lattice [18, 19]. The accuracy of the Ansatz is controlled by the bond dimension, $D$, of the tensors (Methods sec- 
tion). While iPEPS have been applied previously to discuss the ground state of the Shastry-Sutherland model 14. 15, 30, here we apply newly-developed methods [16, 20] for representing the thermal states of the system. We analyse the pure Shastry-Sutherland model, meaning a single 2D layer (inset, Fig. 11); $\mathrm{SrCu}_{2}\left(\mathrm{BO}_{3}\right)_{2}$ is known to have weak and frustrated inter-layer interactions (at most $10 \%$ of $J_{D}$ ), Dzyaloshinskii-Moriya (DM) interactions (3\% 31]) and higher-order further-neighbour interactions, none of which affect the first-order nature of the dimer-plaquette transition.

iPEPS results for $C_{p}(T) / T$ as a function of $J / J_{D}$, presented in Fig. 1k, show the same evolution as in $\mathrm{SrCu}_{2}\left(\mathrm{BO}_{3}\right)_{2}$ under pressure. The broad peaks of the gapped dimer and plaquette states move to lower temperatures on approaching the QPT and narrow to a tall, sharp spike at the critical coupling ratio (Figs.22,d). Figure $1 \mathrm{~d}$ shows that the correlation length grows dramatically around the critical point, but remains small at temperatures below it. Figure 1 illustrates how the average dimer spin-spin correlation, a scalar which serves as an order parameter for the nature of the spin state, jumps discontinuously at $T<T_{c}$ but changes to a smooth function of $J / J_{D}$ at $T \geq T_{c}$, in direct analogy to the density of water molecules.

Our iPEPS results computed with $D=10,14$ and 20 show the same qualitative forms and provide good quantitative convergence (Figs. 22,f) towards the critical coupling ratio of zero-temperature iPEPS [14]. From this we estimate the critical temperature $k_{B} T_{c}=0.039(6) J_{D}$. We work in units of $J_{D}$ because the exact $P$-dependence of the magnetic interactions in $\mathrm{SrCu}_{2}\left(\mathrm{BO}_{3}\right)_{2}$ is subject to further uncertainty; taking a linear extrapolation at constant $J$, with errors provided by alternative estimates [17, a value $J_{D}\left(P_{c}\right) / k_{\mathrm{B}}=77(8) \mathrm{K}$ gives a best estimate of $T_{c}=3.0(6) \mathrm{K}$, in excellent agreement with experiment. By analysing the critical scaling of the discontinuity for $D=20$, we deduce (Fig. $2 \mathrm{~s}$ ) that the exponent is fully consistent with the value $\beta=1 / 8$ expected of a $2 \mathrm{D}$ Ising transition. iPEPS calculations in the critical regime become increasingly challenging at lower temperatures (Methods), and a numerical instability occurs in the dimer-phase regions excluded from Figs. 1 1 -e and 2 -d. However, these regions are readily accessed by a different iPEPS Ansatz [16, and also by working with a finite DM interaction, as shown in Extended Data Fig. ED2. This confirms further that the DM interactions of $\mathrm{SrCu}_{2}\left(\mathrm{BO}_{3}\right)_{2}$ have no effect on the physics of the critical point.

To challenge our interpretation of the critical point, we consider the situation in a finite magnetic field. Because the field has little effect on the gapped dimer and plaquette phases, the physics of the critical point should be essentially unaffected. We have measured the specific heat in fields up to $\mu_{0} H=9 \mathrm{~T}$, and indeed we observe near the critical point (18.2 kbar, Fig. $3 \mathrm{k})$ that the peak remains sharp and shows only very minor field-induced changes. These features are reproduced in detail by our iPEPS results, shown in Fig. 3h for a $J / J_{D}$ ratio very close to the $D=14 \mathrm{QPT}$, which also indicate that the small changes can result simply from not pinpointing the exact critical coupling.

Turning to the $2 \mathrm{~K}$ peak, our 18.2 and $22.0 \mathrm{kbar}$ data (Figs. 3a,b) show that the field causes a strong suppression of both its height and position. However, at $9 \mathrm{~T}$ for $22.0 \mathrm{kbar}$, another peak has emerged that is quite different in shape, becoming tall, sharp and isolated from the broad $4 \mathrm{~K}$ hump, which has almost vanished. This behaviour is also observed above $4 \mathrm{~T}$ in our $26.5 \mathrm{kbar}$ data (Fig. 3r). In Fig. 3p we collect all of these peaks to obtain a clear picture of three key phenomena. First, the $4 \mathrm{~K}$ features remain isolated, changing from sharp to broad with increasing $P$ and $H$ quite independently of the complex action below $2 \mathrm{~K}$. Second, the thermal transition of the plaquette phase, occurring at $2 \mathrm{~K}$ at $18.2 \mathrm{kbar}$, is suppressed to zero by fields of $6 \mathrm{~T}$, even at higher pressures that are expected to stabilize it. Third, the new, sharp peak appears to emerge at finite temperature at higher pressures and fields; we believe this to be the ordering transition of the AF phase, but cannot state definitively whether it is also first-order. Because of the challenges encountered by our iPEPS calculations at very low temperatures, we are not currently able to model these transitions reliably.

Here we comment that similar $C_{p}(T)$ data in zero field have appeared recently [26. These authors did observe the tall, sharp critical-point peak in their 1.9 and $2.1 \mathrm{GPa}$ data (shown only in their Supplemental Materials), but provide no explanation. They also observe the $2 \mathrm{~K}$ peak and ascribe it to a plaquette state (which they assume to be the EP phase, below). At pressures beyond the limits of our study, they argue that the $2 \mathrm{~K}$ feature develops into a signature of the AF phase, but do not find the strong, sharp features we obtain in an applied field (Figs. $3 \mathrm{~b}, \mathrm{c}$ ).

Turning to the theoretical underpinnings of the critical-point phase diagram, NMR 32 and neutron scattering experiments [17] indicate that the low-temperature plaquette phase of $\mathrm{SrCu}_{2}\left(\mathrm{BO}_{3}\right)_{2}$ is not that of the Shastry-Sutherland model, where singlets form in the "empty" plaquettes (EP) of the $J$ lattice, meaning those with no $J_{D}$ dimer (inset, Fig. 1 b), but in the "full" plaquettes (FP). From the sensitive energetic competition between the EP and FP phases [30, it is not surprising that the additional 3D and DM terms in $\mathrm{SrCu}_{2}\left(\mathrm{BO}_{3}\right)_{2}$ could cause this discrepancy, so we expect that the state we observe above $18 \mathrm{kbar}$ and below $2 \mathrm{~K}$ is the $\mathrm{FP}$ phase. The important property of both plaquette phases is their two-fold degenerate ground state (only half of the plaquettes may form singlets), and thus the thermal transition out of the plaquette phase has Ising symmetry. The other important observation is that this transition occurs at 2 $\mathrm{K}$, well below the critical point. 


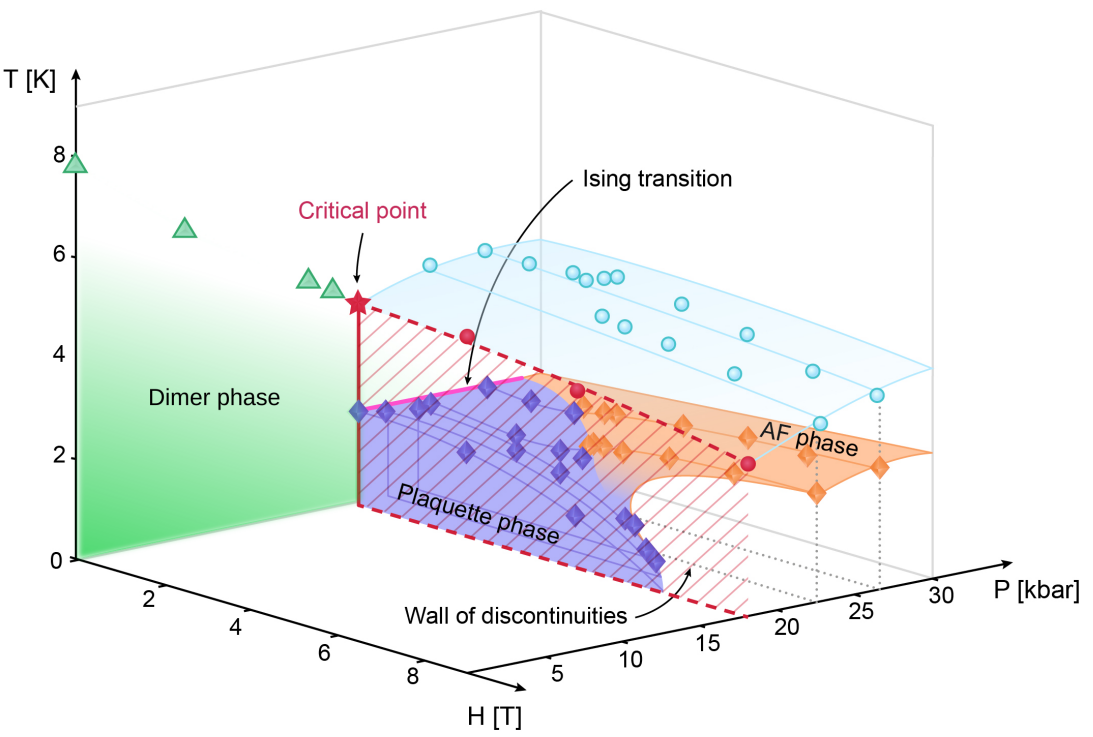

FIG. 4. Schematic phase diagram in field, pressure and temperature. Phase diagram of $\mathrm{SrCu}_{2}\left(\mathrm{BO}_{3}\right)_{2}$ illustrated in the full $(P, H, T)$ space. The turquoise surface and green triangles indicate the locations of the maxima in $C_{p}(T) / T$, respectively in the plaquette and dimer phases. These evolve into the critical point (red star, $\left.T_{c}=3.3 \mathrm{~K}\right)$ in the $(P, T)$ plane. The solid vertical red line below the critical point shows the location of the line of first-order discontinuities at $H=0$; the dashed red lines and red grading mark the wall of discontinuities that extends to finite magnetic field and terminates at critical temperatures (red circles) that fall only slowly with $H$. The horizontal magenta line marks the measured location of the Ising transition out of the plaquette phase at $H=0$. The blue surface marks the volume of parameter space occupied by the plaquette phase: its upper surface is the continuation of the magenta transition line to finite fields and at low pressures it is delimited by the wall of discontinuities. The orange colour indicates the surface of thermal transitions out of the AF phase.
This Ising character contrasts with the fully frustrated bilayer, where the QPT is straight to a $2 \mathrm{D}$ AF phase that by the Mermin-Wagner theorem has no finitetemperature transition, implying that the critical point associated with the discontinuity can only be observed as an isolated point, as in water. In the Shastry-Sutherland model, where the plaquette phase breaks a $\mathrm{Z}_{2}$ symmetry, there are a priori two possibilities for connecting the Ising transition line to the first-order transition line extending from the QPT to the critical point. (i) The Ising transition line ends when it touches the first-order line at a temperature $T<T_{c}$, forming a critical endpoint [27, 33, 34. (ii) The point $\left(P_{c}, T_{c}\right)$ is in fact a tricritical Ising point, where the first-order line turns continuously into the Ising line. Possibility (i) is clearly favoured by our specific-heat data, which show the critical point at $P_{c} \simeq 19 \mathrm{kbar}$ and $T_{c} \simeq 3.3 \mathrm{~K}$ to be well isolated and to exhibit no anomalies around $T_{c}$, while the intrinsic critical temperature of the plaquette phase remains below $2 \mathrm{~K}$.

We represent this situation in the $(P, T)$ plane of Fig.4. On the scale of the figure, the first-order line is essentially vertical in temperature [27. Although a structural transition is probably associated with the magnetic transition in $\mathrm{SrCu}_{2}\left(\mathrm{BO}_{3}\right)_{2}$, causing a discontinuity in the coupling constants, we stress that there is only one transition as a function of $J / J_{D}$ (Fig. 1). In Fig. 4 we have gathered all the features abstracted from our data in field as well as pressure. We find that the picture of the critical point at $H=0$ is supported by the data at finite fields, which indicate a line of critical points, and a wall of first-order transitions, that dominate the $(P, H, T)$ phase diagram. Below $T_{c}(P, H)$, this wall defines a plane through which the average dimer spin-spin correlation should change discontinuously, a prediction that may be tested by measuring the $P$-dependence of the instantaneous spin correlation function by neutron scattering, or of phonon modes sensitive to the magnetic correlations 35$]$.

Below the critical point in Fig. 4, our data reveal a wealth of quantum and thermal phase transitions occurring between 0 and $2 \mathrm{~K}$. The field suppresses the continuous thermal transitions out of the plaquette phase and in its place we have found the AF phase, for the first time at pressures as low as 22.0 kbar. The pressure-induced plaquette-AF QPT is thought to be weakly first-order [14, although it has recently been proposed as a candidate deconfined quantum critical point 36 . While our data in different regimes suggest that the field-induced plaquette-AF QPT could be first- or second-order, there is no clear sign of a second finite-temperature critical point. An experimental verification of the universality classes of these transitions poses a challenge to specificheat measurements under such extreme conditions of pressure, field and temperature. Nevertheless, given the recent numerical and experimental progress in probing the thermal properties of frustrated systems, such verification may soon become possible.

The physics of the critical-point phase diagram appears rather different from that of second-order QPTs: because one may pass from one side of the discontinuity to the other without crossing a transition, there is no breaking of symmetry (here $\mathrm{SU}(2)$ spin). At the critical point itself, the property of a divergent correlation length (Fig. 11d), associated with domain sizes and having critical exponents set by the universality class, remains. The supercritical regime has recently become a 
subject of active investigation, even (with a view to sensitive switching) in critical fluids [37. In the Mott metalinsulator phase diagram, theory [38, 39] and experiment 40 have suggested the emergence of quantum critical scaling in this regime. In $\mathrm{SrCu}_{2}\left(\mathrm{BO}_{3}\right)_{2}$, the striking feature of the phase diagram is that the temperature, $T_{\max }$, characterizing the peak in $C_{p} / T$ reaches a minimum at $T_{c}$. We show in Fig. ED3 that this behaviour is universal around an Ising critical point in 2D lattice models. Thus the specific heat clearly defines not one but two characteristic lines in the supercritical regime (Figs. 1 1 c), in contrast to the correlation length (Fig. 1 d) and the critical isochore (Fig. 1e), both of which are regarded as marking a single crossover line. Although this remarkable property of the specific heat is quite different from water (Fig. 1a), we stress that it is intrinsic to a model as simple as the Ising model. While the origin of this complex physics deserves further theoretical analysis, we observe that both types of behaviour can be probed experimentally in quantum spin systems by comparing the specific heat with scattering measurements of the order parameter.

In summary, we have shown that the first-order QPT in the quantum magnetic material $\mathrm{SrCu}_{2}\left(\mathrm{BO}_{3}\right)_{2}$ is accompanied by a finite-temperature critical point analogous to the phase diagram of water. Our studies span a wide range of pressures and applied magnetic fields around the critical regime and, by revealing the connectivity of the phase diagram and the location of the antiferromagnetic phase, illustrate the importance of controlling the environment to complete studies of criticality and universality. We have explained our experimental data by powerful new numerical methods allowing access to the finite-temperature regime of frustrated systems in two dimensions. As modern quantum magnetism and spintronics embrace spin-orbit coupling and the resulting highly spin-anisotropic interactions required to produce the topological physics of Ising, Kitaev, skyrmion and other systems, a full understanding of the resulting firstorder QPTs will include the quantum phenomenology of the critical point, whose classical variant has been known to science for two centuries.

[1] Cagniard de la Tour, C. Exposé de quelques résultats obtenus par l'action combinée de la chaleur et de la compression sur certains liquides, tels que l'eau, l'alcool, l'éther sulfurique et l'essence de pétrole rectifié, [Presentation of some results obtained by the combined action of heat and compression on certain liquids, such as water, alcohol, sulfuric ether and distilled petroleum spirit]. Annales de chimie et de physique 21, 127 (1822).

[2] Chaikin, P. M. \& Lubensky, T. C. Principles of condensed matter physics (Cambridge University Press, Cambridge, 1995).
[3] Georges, A., Kotliar, G., Krauth, W. \& Rozenberg, M. J. Dynamical mean-field theory of strongly correlated fermion systems and the limit of infinite dimensions. Rev. Mod. Phys. 68, 13 (1996).

[4] Limelette, P. et al. Universality and Critical Behavior at the Mott Transition. Science 302, 89 (2003).

[5] Kagawa, F., Miyagawa, K. \& Kanoda, K. Unconventional critical behaviour in a quasi-two-dimensional organic conductor. Nature 436, 534 (2005).

[6] Sachdev, S. Quantum Phase Transitions (Cambridge University Press, Cambridge, 2011).

[7] Rüegg, C. et al. Quantum Magnets under Pressure: Controlling Elementary Excitations in $\mathrm{TlCuCl}_{3}$. Phys. Rev. Lett. 100, 205701 (2008).

[8] Merchant, P. et al. Quantum and classical criticality in a dimerized quantum antiferromagnet. Nature Phys. 10, 373 (2014).

[9] Giamarchi, T., Rüegg, C. \& Tchernyshyov, O. BoseEinstein condensation in magnetic insulators. Nature Phys. 4, 198 (2008).

[10] Thielemann, B. et al. Direct Observation of Magnon Fractionalization in a Quantum Spin Ladder. Phys. Rev. Lett. 102, 107204 (2009).

[11] $\mathrm{Yu}, \mathrm{R}$. et al. Bose glass and Mott glass of quasiparticles in a doped quantum magnet. Nature 489, 379 (2012).

[12] Shastry, B. S. \& Sutherland, B. Exact ground state of a quantum mechanical antiferromagnet. Physica $B+C$ 108, 1069 (1981).

[13] Miyahara, S. \& Ueda, K. Theory of the orthogonal dimer Heisenberg spin model for $\mathrm{SrCu}_{2}\left(\mathrm{BO}_{3}\right)_{2}$. J. Phys.: Condens. Matter 15, R327 (2003).

[14] Corboz, P. \& Mila, F. Tensor network study of the Shastry-Sutherland model in zero magnetic field. Phys. Rev. B 87, 115144 (2013).

[15] Matsuda, Y. H. et al. Magnetization of $\mathrm{SrCu}_{2}\left(\mathrm{BO}_{3}\right)_{2}$ in Ultrahigh Magnetic Fields up to 118 T. Phys. Rev. Lett. 111, 137204 (2013).

[16] Wietek, A. et al. Thermodynamic properties of the Shastry-Sutherland model throughout the dimer-product phase. Phys. Rev. Res. 1, 033038 (2019).

[17] Zayed, M. E. et al. 4-spin plaquette singlet state in the Shastry-Sutherland compound $\mathrm{SrCu}_{2}\left(\mathrm{BO}_{3}\right)_{2}$. Nature Phys. 13, 962 (2017).

[18] Verstraete, F. \& Cirac, J. I. Renormalization algorithms for quantum-many body systems in two and higher dimensions. arXiv:cond-mat/0407066.

[19] Jordan, J., Orús, R., Vidal, G., Verstraete, F. \& Cirac, J. I. Classical simulation of infinite-size quantum lattice systems in two spatial dimensions. Phys. Rev. Lett. 101, 250602 (2008).

[20] Czarnik, P., Dziarmaga, J. \& Corboz, P. Time evolution of an infinite projected entangled pair state: An efficient algorithm. Phys. Rev. B 99, 035115 (2019).

[21] Witczak-Krempa, W., Chen, G., Kim, Y.-B. \& Balents, L. Correlated Quantum Phenomena in the Strong SpinOrbit Regime. Annu. Rev. Condens. Matter Phys. 5, 57 (2014).

[22] Chacon, A. et al. Observation of two independent skyrmion phases in a chiral magnetic material. Nature Phys. 14, 936 (2018).

[23] Because the first-order line has no critical properties, only its termination point is a "critical point." Following M. E. Fisher 33, 34] we reserve the terminology "critical endpoint" for the termination of a line of continuous (second- 
order) phase transitions 27.

[24] Kageyama, H. et al. Exact dimer ground state and quantized magnetization plateaus in the two-dimensional spin system $\mathrm{SrCu}_{2}\left(\mathrm{BO}_{3}\right)_{2}$. Phys. Rev. Lett. 82, 3168 (1999).

[25] Knetter, C., Bühler, A., Müller-Hartmann, E. \& Uhrig, G. S. Dispersion and symmetry of bound states in the Shastry-Sutherland model. Phys. Rev. Lett. 85, 3958 (2000).

[26] Guo, J. et al. Quantum phases of $\mathrm{SrCu}_{2}\left(\mathrm{BO}_{3}\right)_{2}$ from high-pressure thermodynamics. Phys. Rev. Lett. 124, 206602 (2020).

[27] Stapmanns, J. et al. Thermal Critical Points and Quantum Critical End Point in the Frustrated Bilayer Heisenberg Antiferromagnet. Phys. Rev. Lett. 121, 127201 (2018).

[28] Wessel, S. et al. Thermodynamic properties of the Shastry-Sutherland model from quantum Monte Carlo simulations. Phys. Rev. B 98, 174432 (2018).

[29] Larrea J., J., Martelli, V. \& Rønnow, H. M. Highpressure specific heat technique to uncover novel states of quantum matter. J. Phys. Conf. Ser. 1609, 012008 (2020).

[30] Boos, C. et al. Competition between intermediate plaquette phases in $\mathrm{SrCu}_{2}\left(\mathrm{BO}_{3}\right)_{2}$. Phys. Rev. B 100, 140413(R) (2019).

[31] Nojiri, H., Kageyama, H., Onizuka, K., Ueda, Y. \& Motokawa, M. Direct observation of the multiple spin gap excitations in two-dimensional dimer system $\mathrm{SrCu}_{2}\left(\mathrm{BO}_{3}\right)_{2}$. J. Phys. Soc. Jpn. 68, 2906 (1999).

[32] Waki, T. et al. A Novel Ordered Phase in $\mathrm{SrCu}_{2}\left(\mathrm{BO}_{3}\right)_{2}$ under High Pressure. J. Phys. Soc. Jpn. 76, 073710 (2007).

[33] Fisher, M. E. \& Upton, P. J. Universality and interfaces at critical end points. Phys. Rev. Lett. 65, 2402 (1990).

[34] Fisher, M. E. \& Barbosa, M. C. Phase boundaries near critical end points. I. Thermodynamics and universality. Phys. Rev. B 43, 11177 (1991).

[35] Bettler, S., Stoppel, L., Yan, Z., Gvasaliya, S. \& Zhedulev, Z. Competition between intermediate plaquette phases in $\mathrm{SrCu}_{2}\left(\mathrm{BO}_{3}\right)_{2}$. Phys. Rev. Res. 2, 012010 (2020).

[36] Lee, J. Y., You, Y.-Z., Sachdev, S. \& Vishwanath, A. Signatures of a deconfined phase transition on the ShastrySutherland lattice: Applications to quantum critical $\mathrm{SrCu}_{2}\left(\mathrm{BO}_{3}\right)_{2}$. Phys. Rev. X 9, 041037 (2019).

[37] Maxim, F. et al. Visualization of supercritical water pseudo-boiling at Widom line corossover. Nature Commun. 10, 4114 (2019).

[38] Terletska, H., Vučičević, J., Tanasković, D. \& Dobrosavljević, V. Quantum Critical Transport near the Mott Transition. Phys. Rev. Lett. 107, 026401 (2011).

[39] Eisenlohr, H., Lee, S.-S. B., \& Vojta, M. Mott quantum criticality in the one-band Hubbard model: Dynamical mean-field theory, power-law spectra, and scaling. Phys. Rev. B 100, 155152 (2019).

[40] Furukawa, T., Miyagawa, K., Taniguchi, H., Kato, R. \& Kanoda, K. Quantum criticality of Mott transition in organic materials. Nature Phys. 11, 221-224 (2015).

[41] Kageyama, H., Onizuka, K., Yamauchi, T. \& Ueda, Y. Crystal growth of the two-dimensional spin gap system $\mathrm{SrCu}_{2}\left(\mathrm{BO}_{3}\right)_{2}$. J. Cryst. Growth 206, 65 (1999).

[42] Jorge, G. A. et al. High magnetic field magnetization and specific heat of the $2 \mathrm{D}$ spin-dimer system $\mathrm{SrCu}_{2}\left(\mathrm{BO}_{3}\right)_{2}$. J. Alloys Compd. 369, 90 (2004).
[43] Gmelin, E. Classical temperature-modulated calorimetry: A review. Thermochimica Acta 304-305, 1 (1997).

[44] Nishio, Y., Maeshima, N., Gendiar, A. \& Nishino, T. Tensor product variational formulation for quantum systems. arXix:cond-mat/0401115.

[45] Li, W. et al. Linearized tensor renormalization group algorithm for the calculation of thermodynamic properties of quantum lattice models. Phys. Rev. Lett. 106, 127202 (2011).

[46] Czarnik, P., Cincio, L. \& Dziarmaga, J. Projected entangled pair states at finite temperature: Imaginary time evolution with ancillas. Phys. Rev. B 86, 245101 (2012).

[47] Czarnik, P. \& Dziarmaga, J. Projected entangled pair states at finite temperature: Iterative self-consistent bond renormalization for exact imaginary time evolution. Phys. Rev. B 92, 035120 (2015).

[48] Kshetrimayum, A., Rizzi, M., Eisert, J. \& Orús, R. Tensor network annealing algorithm for two-dimensional thermal states. Phys. Rev. Lett. 122, 070502 (2019).

[49] Jiang, H. C., Weng, Z. Y. \& Xiang, T. Accurate determination of tensor network state of quantum lattice models in two dimensions. Phys. Rev. Lett. 101, 090603 (2008).

[50] Corboz, P., Rice, T. M. \& Troyer, M. Competing states in the $t$ - $J$ model: Uniform $d$-wave state versus stripe state. Phys. Rev. Lett. 113, 046402 (2014).

[51] Nishino, T. \& Okunishi, K. Corner transfer matrix renormalization group method. J. Phys. Soc. Jpn. 65, 891 (1996).

[52] Orús, R. \& Vidal, G. Simulation of two-dimensional quantum systems on an infinite lattice revisited: Corner transfer matrix for tensor contraction. Phys. Rev. B 80, 094403 (2009).

[53] Singh, S., Pfeifer, R. N. C. \& Vidal, G. Tensor network states and algorithms in the presence of a global U(1) symmetry. Phys. Rev. B 83, 115125 (2011).

[54] Bauer, B., Corboz, P., Orús, R. \& Troyer, M. Implementing global Abelian symmetries in projected entangledpair state algorithms. Phys. Rev. B 83, 125106 (2011).

\section{Acknowledgements}

We are grateful to R. Gaal, J. Piatek and M. de Vries for technical assistance. We acknowledge helpful discussions with D. Badrtdinov, C. Boos, T. Fennell, A. Turrini, A. Wietek and A. Zheludev. We thank the São Paulo Research Foundation (FAPESP) for financial support under Grant No. 2018/08845-3, the Qatar Foundation for support through Carnegie Mellon University in Qatar's Seed Research programme, the Swiss National Science Foundation (SNSF) for support under Grant No. 188648 and the European Research Council (ERC) for support under the EU Horizon 2020 research and innovation programme (Grant No. 677061), as well as from the ERC Synergy Grant HERO. We are grateful to the Deutsche Forschungsgemeinschaft for the support of RTG 1995 and to the IT Center at RWTH Aachen University and the JSC Jülich for access to computing time through JARAHPC.

\section{Author contributions}

The experimental project was conceived by H.M.R. and Ch.R. and the theoretical framework was put forward by 
F.M. The crystals were grown by E.P. and K.C. Specificheat measurements were performed by J.L.J. with assistance from M.E.Z., R.L. and H.M.R. S.C. and P.C. performed iPEPS calculations. A.L. performed complementary exact diagonalization calculations. L.W. and S.W. performed quantum Monte Carlo calculations on the fully frustrated bilayer model. Data analysis and figure preparation were performed by J.L.J., E.F., S.C., L.W., S.W., P.C. and H.M.R. The detailed theoretical analysis was provided by P.C., S.C., F.M., A.H., B.N., L.W. and S.W. The manuscript was written by B.N. and F.M. with assistance from all the authors.

\section{Additional information}

The authors declare no competing financial interests. The statements made herein are not the responsibility of the Qatar Foundation. Correspondence and requests for materials should be addressed to H.M.R. (henrik.ronnow@epfl.ch).

\section{Methods Section and Extended Data Figures for}

\section{A quantum magnetic analogue to the critical point of water}

J. Larrea Jiménez, S. P. G. Crone, E. Fogh, M. E. Zayed, R. Lortz, E. Pomjakushina, K. Conder, A. M. Läuchli, L. Weber, S. Wessel, A. Honecker, B. Normand, Ch. Rüegg, P. Corboz, H. M. Rønnow and F. Mila,

\section{Methods}

Samples. Single crystals of $\mathrm{SrCu}_{2}\left(\mathrm{BO}_{3}\right)_{2}$ were grown by a floating-zone method. First polycrystalline $\mathrm{SrCu}_{2}\left(\mathrm{BO}_{3}\right)_{2}$ was prepared by a solid-state reaction using as starting materials $\mathrm{SrCO}_{3}, \mathrm{CuO}$ and $\mathrm{B}_{2} \mathrm{O}_{3}$ with 99.99\% purity. These were mixed, ground and heattreated at $900^{\circ} \mathrm{C}$ in flowing oxygen for over $100 \mathrm{~h}$ with several intermediate grindings. The phase purity was verified by conventional $x$-ray diffractometry and the powder was pressed hydrostatically into rods $(8 \mathrm{~mm}$ in diameter and around $90 \mathrm{~mm}$ in length) which were sintered at $900^{\circ} \mathrm{C}$ for $20 \mathrm{~h}$ in an oxygen atmosphere.

Single-crystal growth was carried out using an Optical Floating Zone Furnace (FZ-T-10000-H-IV-VP-PC, Crystal System Corp., Japan) with four $300 \mathrm{~W}$ halogen lamps as the heat source. Although the first reported single crystals of $\mathrm{SrCu}_{2}\left(\mathrm{BO}_{3}\right)_{2}$ were also grown in this type of furnace, using $\mathrm{LiBO}_{2}$ discs as a solvent placed on a top of a seed rod [41, we obtained better results using a selfadjusted flux method [42. Optimal growth conditions were found to be a steady growth rate of $0.25 \mathrm{~mm} / \mathrm{h}$ at all times, rotation of both feeding and seeding rods at approximately $15 \mathrm{rpm}$ in opposite directions to ensure homogeneity of the liquid and the application of a 5 bar pressure of argon with $20 \%$ oxygen. When a homogeneous melt was achieved, the power of the lamps was decreased slowly (over 24 hours) until steady-state conditions were found (c. $48.5 \%$ lamp power in the present case), after which these conditions were maintained rigorously until the end of the growth. The full growth process required approximately two weeks and resulted in a recrystallized boule of $8 \mathrm{~cm}$ in length containing a single-crystalline grain in its top $4 \mathrm{~cm}$.

Specific-heat measurements. Three slabs were cut from the single-crystal rod, each with a cross-section of $7.6-9.0 \mathrm{~mm}^{2}$ in the $a b$ plane and a thickness of $0.5-1.0 \mathrm{~mm}$ parallel to the $c$-axis. The sample masses were measured to be between 15 and $36 \mathrm{mg}$. The samples were polished gently to deposit $\mathrm{Pt}$ films of $20 \mathrm{~nm}$ thickness. To measure the heat capacity, $C(P, H, T)$, under multiple extreme conditions (very low temperatures, high pressures and intense magnetic fields), we used alternatingcurrent (a.c.) calorimetry at a second-harmonic mode (" $2 \omega$ "). We employed a piston cylinder BeCu clamp cell to apply pressures up to $26.5 \mathrm{kbar}$, with liquid kerosene as the pressure-transmitting medium. Measurements of the electrical resistivity at the superconducting transition of a $\mathrm{Pb}$ strip were used as a pressure manometer and revealed good hydrostatic conditions in our experiments. The pressure cell was inserted into a cryostat with a ${ }^{3} \mathrm{He}^{4} \mathrm{He}$ dilution refrigerator that allowed both cell and sample to be cooled to $0.1 \mathrm{~K}$ with temperature variations below $1 \mathrm{mK}$. At some pressure steps, a magnetic field up to $9 \mathrm{~T}$ was applied using a superconducting magnet whose field direction was perpendicular to the $c$ axis.

Our a.c. calorimetry set-up was sputtered onto the sample slab to guarantee good thermal contact of the sample, heater and thermometer [29]. It consisted of two separate Pt films, one used as the heater and the other used to improve the thermal contact between sample and thermometer, as shown in Fig. ED1 1 . This configuration allowed the efficient measurement of the modulated temperature difference in the sample $\left(\Delta T_{a c}\right)$ as a function of the amplitude and frequency, $f=\omega / 2 \pi$, of the alternating excitation current, $I_{\mathrm{ex}}=I_{0} e^{-i \omega t}$. The heating power was applied through two Constantan wires $\left(\mathrm{H}_{1 \mathrm{a}}\right.$ and $\mathrm{H}_{1 \mathrm{~b}}$ in Fig. ED1 1 ), this choice of metal being made to avoid heat leakage through the wires; the gold wires $\left(\mathrm{H}_{2 \mathrm{a}}\right.$ and $\mathrm{H}_{2 \mathrm{~b}}$ ) were applied to measure the electrical resistance of the heater as a function of $P, H$ and $T$. We used a 
a
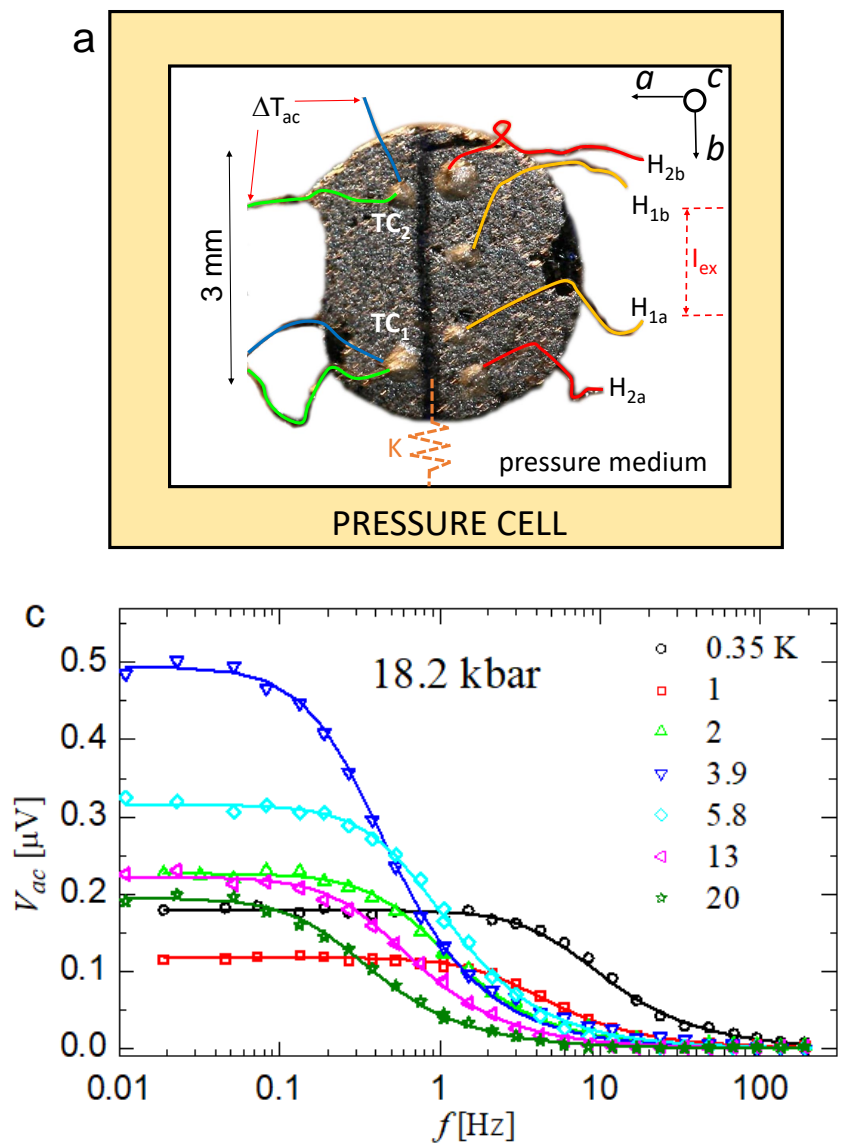

b
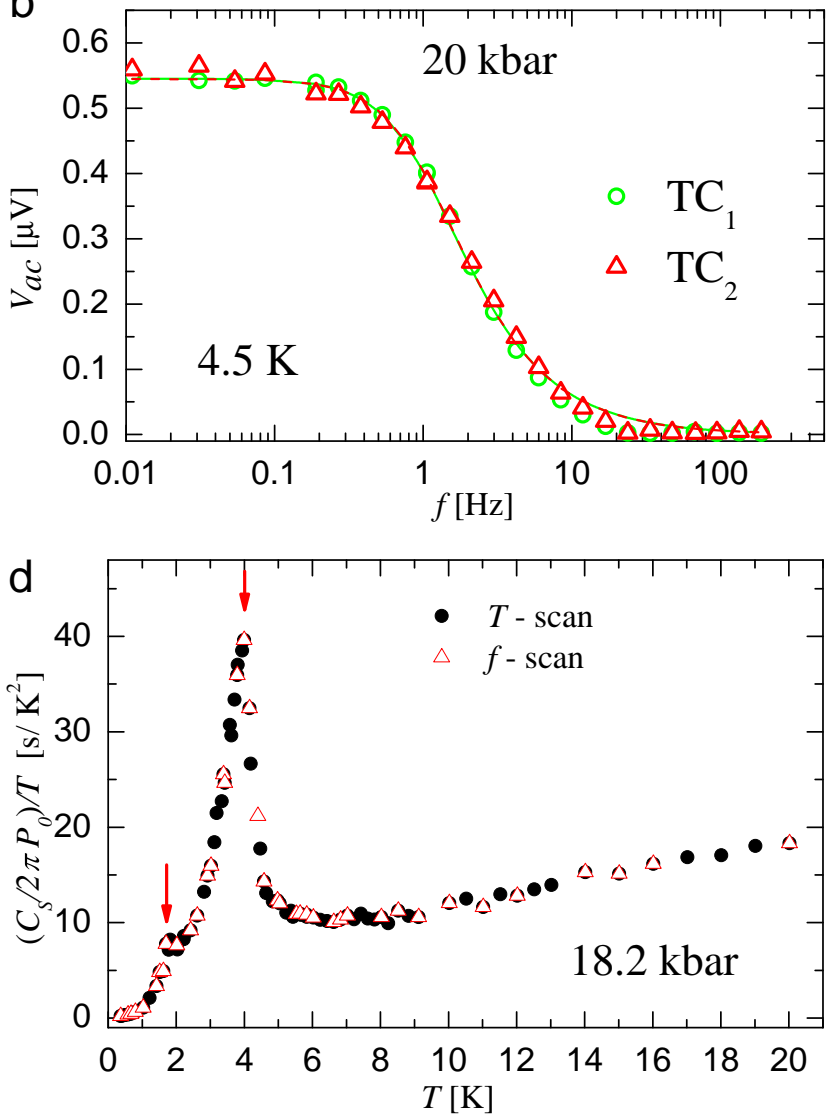

FIG. ED1. a.c. calorimetry on $\mathrm{SrCu}_{2}\left(\mathbf{B O}_{3}\right)_{2}$. a, The a.c. calorimeter was prepared by depositing two Pt thin films (the shinier surfaces) over both halves of the sample. One film was used as the heater and the other for optimal thermal contact and measurement. The heating current $\left(I_{\mathrm{ex}}\right.$ at frequency $f$ ) was supplied through the pair of Constantan wires, $\mathrm{H}_{1 \mathrm{a}}$ and $\mathrm{H}_{1 \mathrm{~b}}$, while $\mathrm{H}_{2 \mathrm{a}}$ and $\mathrm{H}_{2 \mathrm{~b}}$ were used to measure the electrical resistance, $R_{\mathrm{Pt}}$, of the Pt film. $\mathrm{TC}_{1}$ and $\mathrm{TC}_{2}$ are thermocouples and $K$ represents the thermal contact between the sample and the cryostat (through the pressure cell). $\mathbf{b}$, Isothermal $(T=4.5$ $\mathrm{K})$ and isobaric $(P=20 \mathrm{kbar}) f$-dependence of the modulated pick-up voltage, $V_{a c}$, which is directly proportional to the temperature differential, $\Delta T_{a c}$, measured by the thermocouples at two different positions. c, Isobaric $(P=18.2 \mathrm{kbar}) f$ dependence measurements of $V_{a c}$ at different temperatures with $I_{0}=1.6 \mathrm{~mA}$ at $T \geq 3.9 \mathrm{~K}, I_{0}=0.8 \mathrm{~mA}$ at $T=2 \mathrm{~K}$ and $I_{0}=0.4 \mathrm{~mA}$ at $T<2 \mathrm{~K}$. d, Sample heat capacity normalized to the input heating power $\left(P_{0}=I_{0}^{2} R_{\mathrm{Pt}}\right)$, comparing the fit of $V_{a c}(f)$ obtained from the steady-state equation (" $f$-scan" 29]) with values obtained directly from a variable-temperature measurement performed at the fixed working frequency $f_{C}=1.5 \mathrm{~Hz}$ ("T-scan"). Solid and dashed lines in panels $\mathbf{b}$ and $\mathbf{c}$ represent fits using the steady-state equation [29, 43].

$\mathrm{AuFe}(0.07) /$ Chromel thermocouple $\left(\mathrm{TC}_{2}\right.$ in Fig. ED1 $)$ as the thermometer detecting the temperature differential, $\Delta T_{a c}$, between the sample and its environment, and placed a second thermocouple $\left(\mathrm{TC}_{1}\right)$ symmetrically opposite to it; because $\mathrm{TC}_{1}$ showed the same $f$-dependence of $\Delta T_{a c}$ as $\mathrm{TC}_{2}$, we could conclude that there were no thermal gradients across the sample within the resolution of our experiment.

The sample heat capacity was obtained directly from the isothermal $f$-scans, shown in Figs. ED1p-c, by fitting with the standard steady-state equation [43]. Given the complete control of all relevant a.c. calorimetry parameters, our method has a number of advantages in the determination of absolute values of $C(P, H, T)$ when com- pared with previous work [26]. These advantages include a more precise determination of the corrections for unwanted heat losses throughout the sample, a more precise separation of additional $P$-dependent contributions and the ability to achieve an optimal thermal equilibrium inside the sample (within the resolution of the thermocouple). Thus, our " $f$-scan" analysis [29] allows an accurate determination of the working frequency $\left(f_{C}\right)$ at which to maximize $C(P, H, T)$ at each pressure. Figures ED1k-d demonstrate the correct determination of $f_{C}=1.5 \mathrm{~Hz}$ at $P=18.2$ kbar, whose measurement at fixed $f_{C}$ (a " $T$ scan") reproduces the same result as obtained from our $f$-scan analysis 29$]$.

For practical purposes in our measurements of 

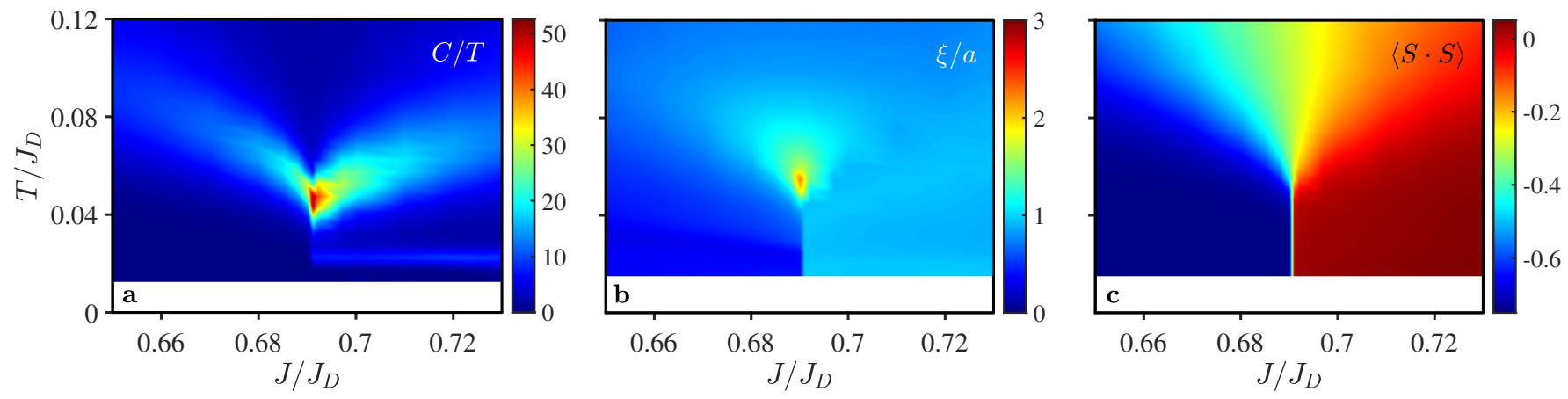

FIG. ED2. The critical point in the presence of Dzyaloshinskii-Moriya interactions. Thermodynamic data obtained from iPEPS calculations with $D=10$ performed for the Shastry-Sutherland model in the presence of Dzyaloshinskii-Moriya interactions. These interactions are placed on the dimer $\left(J_{D}\right)$ bonds and have the magnitude $\left(D_{D} / J_{D}=0.03\right)$ known for $\mathrm{SrCu}_{2}\left(\mathrm{BO}_{3}\right)_{2}$. They create an entangled ground state in the dimer phase, which resolves the numerical instabilities observed for the pure Shastry-Sutherland model at low temperatures, although the reduced symmetry limits the maximum $D$ to 10 . a, Specific heat, $C_{p}\left(J / J_{D}, T\right) / T$, shown in the same format as for Figs. 1b-c. b, Correlation length, $\xi$, showing clearly the region of "pressure" and temperature over which Ising correlations develop. c, Dimer spin-spin correlation function, $\left\langle\vec{S}_{i} \cdot \vec{S}_{j}\right\rangle$, emphasizing the abrupt onset with decreasing temperature of a sharp discontinuity as a function of $J / J_{D}$. It is clear that these Dzyaloshinskii-Moriya interactions have no qualitative effect whatsoever on the physics of the critical point.

$\mathrm{SrCu}_{2}\left(\mathrm{BO}_{3}\right)_{2}$, we found that the range of pressures and fields covered by our current investigation had negligible influence on the relevant parameters in the steady-state equation. Thus we measured the $T$-dependence of the heat capacity of each sample at a constant field and at a fixed frequency, $f_{C}$, determined for each pressure. Our methodology allowed us to determine the heat capacity within an accuracy of $5 \%$ with respect to an adiabatic technique. Further details concerning all aspects of the procedures of our a.c. calorimetry measurements may be found in Ref. [29].

iPEPS. Tensor-network methods provide a powerful approach for accurate numerical calculations of both the ground and thermal states of gapped local Hamiltonians. iPEPS [18, 19, 44, a two-dimensional generalization of matrix-product states, are a variational Ansatz allowing both wavefunctions and thermal states [20, 45, 48, to be represented efficiently in the thermodynamic limit, with the accuracy of the representation controlled systematically by the bond dimension, $D$, of the tensors.

To obtain an iPEPS representation of a thermal state, we employ the algorithms of Ref. 20, which are based on the imaginary-time evolution of a purification of the thermal density operator. At each time step the bond dimension of the iPEPS is truncated to the maximal $D$. To maximize $D$ while working near the QPT, we restrict the truncation to the (local) simple-update approach [16, 49]. Physical expectation values are computed by contracting the tensor network, which we perform by a development [50] of the corner-transfer-matrix method [51, 52]. This method is also used to compute the transfer matrix, from which we extract the correlation length, $\xi$ in Figs. 1d and ED2 $b$, of the correlation function with the slowest decay. To improve the efficiency of the calculations we exploit the global U(1) symmetry of the model [53, 54, which for a pure Heisenberg model is preserved in the presence of an applied field at finite temperatures.

In the Shastry-Sutherland problem, the optimal tensor geometry depends on the ground state [14]: the spin correlations of the dimer phase are represented most efficiently using one tensor per dimer in a two-dimer unit cell [16, while correlations in the plaquette phase are best described by an Ansatz with one tensor per four sites (from four different dimers) on a plaquette [14. For working around a critical point, it is essential to use a single representation of both quantum phases, and in the Shastry-Sutherland model the plaquette basis is more efficient for representing the dimer phase than the reverse. For this reason, we have used the plaquette basis for all of the results reported here.

When working in the dimer phase, we do observe numerical instabilities at low temperatures, which are most probably an artifact arising due to the product nature of the dimer ground state, as all observables and energies are essentially converged at those temperatures. One means of circumventing this problem is to add a small Dzyaloshinskii-Moriya interaction to the model, in fact guided by the real spin Hamiltonian of $\mathrm{SrCu}_{2}\left(\mathrm{BO}_{3}\right)_{2}$. This leads to an entangled ground state, which as shown in Fig. ED2 makes the low-temperature regime accessible, albeit at cost of a reduced $D$.

When working in the plaquette phase, the strongly entangled nature of the plaquette ground state makes lowtemperature studies very challenging, and for this reason our primary focus is on temperatures $T / J_{D} \gtrsim 0.03$, for a full characterization of the regime relevant to the critical point. Although a small feature is evident in $C / T$ around $T / J_{D}=0.02$ (Fig. ED2 2 ), we caution against associat- 

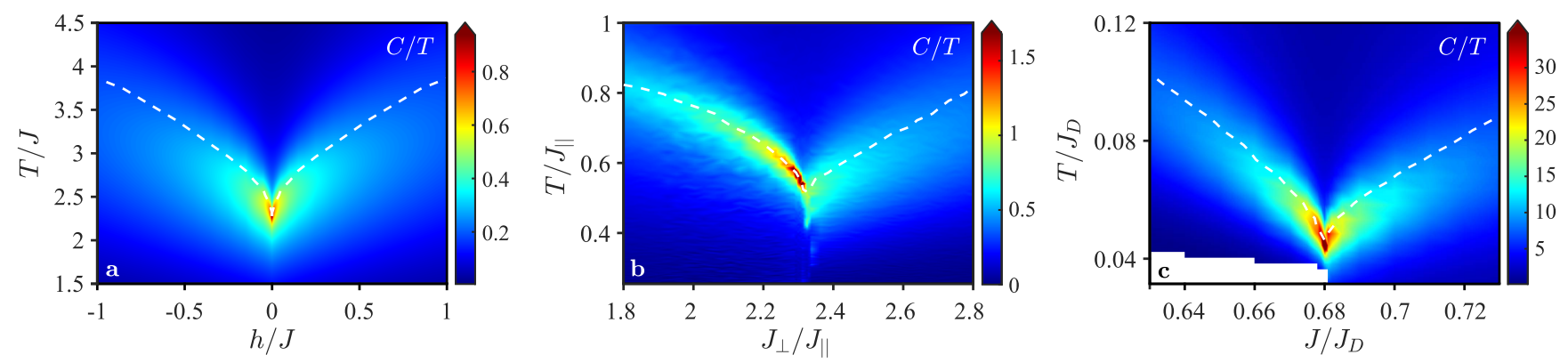

FIG. ED3. Ising critical points in different lattice models. Specific heat, $C / T$, for a number of $2 \mathrm{D}$ models, illustrating its universal behaviour around the Ising critical point. a, Ising model on the square lattice in a longitudinal magnetic field, $h$, obtained by contracting the exact $D=2$ tensor-network representation of the partition function using the CTM method with a boundary bond dimension $\chi=24$ 51. b, Fully frustrated bilayer model, obtained by using the stochastic series expansion quantum Monte Carlo approach developed in Refs. 27, 28, to perform simulations on systems of sizes up to $2 \times 32 \times 32$ as a function of $J_{\perp} / J_{\|}$. c, Shastry-Sutherland model, obtained by iPEPS calculations with $D=20$ as in Fig. 1c. The dashed lines show the positions of the local maxima of the specific heat, $C\left(J / J_{D}\right)$, which we label by their temperatures, $T_{\max }$. These two lines reach an absolute minimum, $T_{\max }=T_{c}$, where they meet at the Ising critical point, with $T_{\max }$ increasing as the control parameter is changed away from the QPT. Thus the specific heat defines two characteristic lines in the phase diagram of the Ising critical point, rather than the single line given by the correlation length (Fig. 1d) and the critical isochore (Fig. 1e). We stress that this contrast is a fundamental property of the Ising model, and hence of all models sharing its physics. For the Shastry-Sutherland model (c), the two lines of maxima could be taken to provide a qualitative definition of "dimer-like" and "plaquette-like" regimes, accompanied by a third regime bearing no clear hallmarks of either $T=0$ phase. We remark that the values of $T_{c}$ in units of the relevant energy scale, $T_{c} / J=2.3(\mathbf{a}), T_{c} / J_{\|}=0.53(\mathbf{b})$ and $T_{c} / J_{D}=0.04$ (c), vary widely among the three models. This can be traced to the change in slope of the ground-state energy at the transition, whose compensation by entropy effects restores a derivable free energy at $T_{c}$.

ing this with the Ising transition of the plaquette phase, because we do not find corresponding behaviour in the spin correlations and thus cannot confirm the reliability of our results at such low temperatures. 